\section{(6) OPEN ACCESS}

\title{
Efficacy and safety of natalizumab in multiple sclerosis: interim observational programme results
}

\author{
Helmut Butzkueven, ${ }^{1,2}$ Ludwig Kappos, ${ }^{3}$ Fabio Pellegrini, ${ }^{4}$ Maria Trojano, ${ }^{5}$ \\ Heinz Wiendl, ${ }^{6}$ Radhika N Patel, ${ }^{7}$ Annie Zhang, ${ }^{7}$ Christophe Hotermans, ${ }^{7}$ \\ Shibeshih Belachew, ${ }^{7}$ on behalf of the TYSABRI Observational Program (TOP) \\ Investigators
}

\section{- Additional material is published online only. To view please visit the journal online (http://dx.doi.org/10.1136/ jnnp-2013-306936). \\ For numbered affiliations see end of article. \\ Correspondence to Dr H Butzkueven, \\ Department of Medicine, Royal Melbourne Hospital Brain \\ Centre, University of Melbourne, Level 4 East, Royal Melbourne Hospital Campus, Grattan Street, Parkville, Melbourne, VIC 3050 Australia; \\ butz@unimelb.edu.au}

Received 3 October 2013 Revised 20 December 2013 Accepted 27 December 2013 Published Online First 14 February 2014

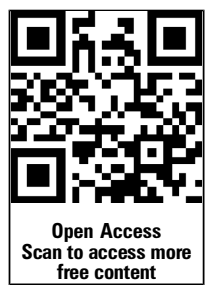

\section{Linked}

- http://dx.doi.org/10.1136/ jnnp-2013-307355

\section{CrossMark}

\footnotetext{
To cite: Butzkueven $\mathrm{H}$, Kappos L, Pellegrini F, et al. J Neurol Neurosurg Psychiatry 2014;85: 1190-1197.
}

\section{ABSTRACT}

Background Clinical trials established the efficacy and safety of natalizumab. Data are needed over longer periods of time and in the clinical practice setting.

Objective To evaluate long-term safety of natalizumab and its impact on annualised relapse rate and Expanded Disability Status Scale (EDSS) progression in patients with relapsing-remitting multiple sclerosis (RRMS).

Methods The Tysabri (natalizumab) Observational Program (TOP) is an open-label, multinational, 10-year prospective study in clinical practice settings.

Results In this 5-year interim analysis, 4821 patients were enrolled. Follow-up for at least 4 years from natalizumab commencement in 468 patients and at least 2 years in 2496 patients revealed no new safety signals. There were 18 cases of progressive multifocal leucoencephalopathy reported, following 11-44 natalizumab infusions. Mean annualised relapse rate decreased from 1.99 in the 12 months prior to baseline to 0.31 on natalizumab therapy $(p<0.0001)$, remaining low at 5 years. Lower annualised relapse rates were observed in patients who used natalizumab as first MS therapy, in patients with lower baseline EDSS scores, and in patients with lower prenatalizumab relapse rates. Mean EDSS scores remained unchanged up to 5 years. Conclusions Interim TOP data confirm natalizumab's overall safety profile and the low relapse rate and stabilised disability levels in natalizumab-treated patients with RRMS in clinical practice.

Trial registration number NCT00493298.

\section{INTRODUCTION}

Natalizumab (Tysabri, Biogen Idec Inc, Cambridge, Massachusetts, USA) is a selective adhesion molecule inhibitor that blocks $\alpha 4$ integrin, which is expressed on the surface of lymphocytes and is required for endothelial adhesion, facilitating migration of peripheral blood lymphocytes into the central nervous system. ${ }^{1}$

In the 2 -year, phase 3 AFFIRM study ${ }^{2}$ of patients with relapsing-remitting multiple sclerosis (RRMS), natalizumab monotherapy demonstrated consistent efficacy in the overall study population and across multiple subgroups of patients predefined on the basis of demographic and baseline disease characteristics, including age, sex, number of brain MRI lesions, disability status and number of relapses in the prior year. ${ }^{2}{ }^{3}$
While the AFFIRM trial established the safety and efficacy of natalizumab, data are needed to confirm the safety and efficacy of natalizumab over treatment durations longer than 2 years and, importantly, in a clinical practice setting.

The Tysabri (natalizumab) Observational Program (TOP) was designed to evaluate the longterm safety of natalizumab monotherapy, as well as its impact on disease activity and disability progression, in patients with RRMS in the clinical practice setting. This paper presents findings from an interim analysis of TOP data from study initiation in July 2007 to a data lock on 1 December 2012.

\section{METHODS}

Patients

Eligible RRMS patients met criteria for natalizumab prescription in their respective countries and had three or fewer natalizumab infusions before enrolment in TOP. Female participants were postmenopausal, surgically sterile, or willing to practice effective contraception.

Patients were enrolled as soon as possible after deciding to start natalizumab treatment. The latest permissible enrolment date was the day before patients' fourth natalizumab infusion (ie, 16 weeks after the start of natalizumab treatment).

The protocol was approved by each centre's independent ethics committee. The study was performed in accordance with the Declaration of Helsinki and Good Clinical Practice guidelines. All patients provided written informed consent.

\section{Study design}

TOP is an ongoing, open-label, multinational, multicentre, prospective, observational study conducted in clinical practice settings in Europe, Australia, Canada and Argentina. Patients receive natalizumab $300 \mathrm{mg}$ intravenously over approximately $1 \mathrm{~h}$, every 4 weeks.

\section{Endpoints and assessments}

Data were collected at regular clinical visits every 6 months. Data entry was Web based. Patients who discontinued natalizumab were encouraged to remain in the study; if patients exited the study for any reason, physicians were asked to collect data on serious adverse events for an additional 6 months where possible. 
The primary endpoint is long-term safety (incidence and type of serious adverse events). Secondary endpoints include measures of MS disease activity (ie, the occurrence of clinical relapses) and change in Expanded Disability Status Scale (EDSS) score. Multiple Sclerosis Severity Scale ${ }^{4}$ scores were recorded at baseline.

A clinical relapse was defined as new or recurrent neurological symptoms, not associated with fever, lasting for $\geq 24 \mathrm{~h}$ and followed by a period of 30 days of stability or improvement. New or recurrent neurological symptoms that occurred $<30$ days following the onset of a protocol-defined relapse were considered part of the same relapse.

Confirmed EDSS progression was defined per protocol as an increase of $\geq 1.0$ point in EDSS score sustained for 6 months.
We also performed analyses comparable to those used in recent clinical trials, in which confirmed EDSS progression was defined as an increase, sustained for 6 months, of $\geq 0.5$ points from a baseline EDSS score $\geq 6.0$, of $\geq 1.0$ point from a baseline EDSS score of $\geq 1.0$ to $<6.0$ or of $\geq 1.5$ points from a baseline EDSS score of $0.0 .^{2} 5$ Confirmed EDSS improvement was assessed in the overall population and in patients with baseline EDSS scores $\geq 2.0$ and was defined as a decrease of $\geq 1.0$ point in EDSS score sustained for 6 months. ${ }^{6}$

Medical review of each case report was performed at the end of data entry. Serious adverse events were evaluated for possible clinical significance or indication of a previously unknown risk. The Drug Safety Department at Biogen Idec evaluated serious adverse event reports to ensure case validity and confirm

Table 1 Baseline characteristics

\begin{tabular}{|c|c|c|c|}
\hline Characteristics & TOP $(\mathrm{N}=4821)$ & AFFIRM overall ( $\mathrm{N}=942)$ & AFFIRM natalizumab $(n=627)$ \\
\hline \multicolumn{4}{|c|}{ A. Baseline characteristics of patients currently enrolled in TOP, compared with AFFIRM patients } \\
\hline Mean age, years (SD) & $37.2(9.69)$ & $36.0(8.3)$ & $35.6(8.5)$ \\
\hline Female, $\mathrm{n}(\%)$ & $3466(72)$ & $660(70)$ & $449(72)$ \\
\hline Mean number of relapses in prior year (SD) & $1.99(1.03)$ & $1.52(0.86)$ & $1.53(0.91)$ \\
\hline \multicolumn{4}{|l|}{ Number of relapses in prior year, $\mathrm{n}(\%)$} \\
\hline$\leq 1$ & $1713(36)$ & $560(59)$ & $374(60)$ \\
\hline$>1$ & $3108(64)$ & $382(41)$ & $253(40)$ \\
\hline EDSS score, mean (SD) & $3.5(1.62) \dagger$ & $2.3(1.2)$ & $2.3(1.2)$ \\
\hline \multicolumn{4}{|l|}{ EDSS score, $n(\%)$} \\
\hline $0.0-2.0$ & $1297(27)$ & $510(54)$ & $339(54)$ \\
\hline $2.5-4.0$ & $2114(44)$ & $369(39)$ & $246(39)$ \\
\hline $4.5-9.5$ & $1386(29)$ & $63(7)$ & $42(7)$ \\
\hline \multicolumn{4}{|c|}{ B. Additional baseline characteristics of patients currently enrolled in TOP } \\
\hline \multicolumn{4}{|c|}{ EDSS score, $n(\%)^{*}$} \\
\hline$<3.0$ & $1773(37)$ & & \\
\hline$\geq 3.0$ & $3024(63)$ & & \\
\hline MSSS score, $† \ddagger$ mean (SD) & $4.97(2.40)$ & & \\
\hline \multicolumn{4}{|l|}{ Prior treatment } \\
\hline Yes, n (\%) & $4384(90.9)$ & & \\
\hline \multicolumn{4}{|l|}{ Prior number of DMTs used, $\mathrm{n}(\%)$} \\
\hline 0 DMT & $437(9)$ & & \\
\hline $1 \mathrm{DMT}$ & $2213(46)$ & & \\
\hline$\geq 2$ DMTs & $2171(45)$ & & \\
\hline \multicolumn{4}{|l|}{ Treatment history, §ণ n (\%) } \\
\hline Therapy naïve & $437(10)$ & & \\
\hline IFN only & $2131(47)$ & & \\
\hline GA only & $421(9)$ & & \\
\hline Switched between GA and IFN & $855(19)$ & & \\
\hline Prior IS use $\mathrm{e}^{\star *}$ & $697(15)$ & & \\
\hline \multicolumn{4}{|l|}{ Disease durationt† } \\
\hline Median duration, years (range) & $7.3(0.0-43.9)$ & & \\
\hline Duration $\geq 8$ years, $\mathrm{n}(\%)$ & $2203(46)$ & & \\
\hline \multicolumn{4}{|l|}{ Treatment duration } \\
\hline Median duration, years (range) & $3.0(0.0-26.5)$ & & \\
\hline Mean duration, years (SD) & $4.1(3.71)$ & & \\
\hline Duration $\geq 3$ years, $n(\%)$ & $2435(51)$ & & \\
\hline \multicolumn{4}{|c|}{ 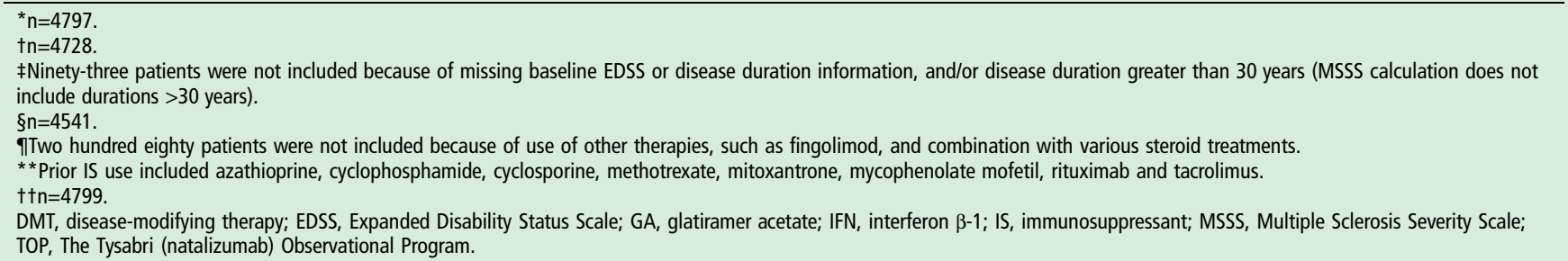 } \\
\hline
\end{tabular}


Table 2 Patient enrolment and disposition

\begin{tabular}{|c|c|}
\hline Number of patients enrolled in TOP & 4821 \\
\hline \multicolumn{2}{|l|}{ Enrolment by number of prior natalizumab doses, $\mathrm{n}(\%)$} \\
\hline 0 & $2058(42.7)$ \\
\hline 1 & $1089(22.6)$ \\
\hline 2 & $875(18.1)$ \\
\hline 3 & 799 (16.6) \\
\hline \multicolumn{2}{|l|}{ Enrolment by country, $n(\%)$} \\
\hline Germany (130 sites) & $1430(29.7)$ \\
\hline Czech Republic (15 sites) & $644(13.4)$ \\
\hline Belgium (34 sites) & $563(11.7)$ \\
\hline Italy (17 sites) & $314(6.5)$ \\
\hline Canada (19 sites) & $291(6.0)$ \\
\hline The Netherlands (22 sites) & $268(5.6)$ \\
\hline Norway (13 sites) & $200(4.1)$ \\
\hline France (65 sites) & $188(3.9)$ \\
\hline Finland (7 sites) & $183(3.8)$ \\
\hline Australia (12 sites) & $179(3.7)$ \\
\hline Slovakia (5 sites) & $141(2.9)$ \\
\hline Greece (12 sites) & $129(2.7)$ \\
\hline Great Britain (14 sites) & $119(2.5)$ \\
\hline Spain (13 sites) & $99(2.1)$ \\
\hline Portugal (8 sites) & $67(1.4)$ \\
\hline Argentina (3 sites) & $6(0.1)$ \\
\hline Number of patients dosed with natalizumab, $\mathrm{n}(\%)$ & $4821(100)$ \\
\hline Number of patients who discontinued natalizumab, ${ }^{*} \mathrm{n}(\%)$ & $1222(25.3)$ \\
\hline \multicolumn{2}{|l|}{ Reasons for natalizumab discontinuation, $\uparrow \mathrm{n}(\%)$} \\
\hline Anti-JCV antibody positive & $277(5.7)$ \\
\hline Medication change $\ddagger$ & $247(5.1)$ \\
\hline Insufficient efficacy & $229(4.8)$ \\
\hline Patient decision & $171(3.5)$ \\
\hline Withdrawal of consent & $142(2.9)$ \\
\hline Adverse event (non-serious adverse event) & $107(2.2)$ \\
\hline Physician decision & $97(2.0)$ \\
\hline Pregnancy/pregnancy desire & $74(1.5)$ \\
\hline Tolerability problem & $65(1.3)$ \\
\hline Natalizumab treatment duration concern & $65(1.3)$ \\
\hline Serious adverse event & $51(1.1)$ \\
\hline Antibodies to natalizumab & $44(0.9)$ \\
\hline Other reason & $35(0.7)$ \\
\hline Non-compliance & $31(0.6)$ \\
\hline Prior IS use & $28(0.6)$ \\
\hline Safety concern & $26(0.5)$ \\
\hline Lost to follow-up & $24(0.5)$ \\
\hline PML§ & $7(0.1)$ \\
\hline Malignancy/cancer & $7(0.1)$ \\
\hline Moved out of area & $5(0.1)$ \\
\hline Inconvenience & $5(0.1)$ \\
\hline Other serious infection & $4(<0.1)$ \\
\hline No reason given in data & $3(<0.1)$ \\
\hline Death & $2(<0.1)$ \\
\hline Opportunistic infection & $1(<0.1)$ \\
\hline Number of patients who withdrew from TOP† & $740(15.3)$ \\
\hline \multicolumn{2}{|l|}{ Reasons for withdrawal from TOP, $\mathrm{n}(\%)$} \\
\hline Lost to follow-up & $121(2.5)$ \\
\hline Medication change $\ddagger$ & $116(2.4)$ \\
\hline Patient decision & $115(2.4)$ \\
\hline Withdrawal of consent & $110(2.3)$ \\
\hline Moved out of the area & $99(2.1)$ \\
\hline Anti-JCV antibody & $62(1.3)$ \\
\hline Physician decision & $52(1.1)$ \\
\hline
\end{tabular}

Table 2 Continued

\begin{tabular}{lc}
\hline Number of patients enrolled in TOP & 4821 \\
\hline Insufficient efficacy & $46(1.0)$ \\
Adverse event (non-serious adverse event) & $43(0.9)$ \\
Inconvenience & $39(0.8)$ \\
Serious adverse event & $34(0.7)$ \\
Other reason & $32(0.7)$ \\
Pregnancy/pregnancy desire & $27(0.6)$ \\
Natalizumab antibody positive & $20(0.4)$ \\
Non-compliance & $13(0.3)$ \\
Safety concern & $11(0.2)$ \\
Tolerability problem & $8(0.2)$ \\
Prior IS use & $8(0.2)$ \\
Death & $2(<0.1)$ \\
PML & $2(<0.1)$ \\
Other serious infection & $2(<0.1)$ \\
No reason given in data & $2(<0.1)$ \\
Malignancy/cancer & $1(<0.1)$ \\
\hline * Of the 1222 patients who discontinued natalizumab, 223 (18.3\%) discontinued after \\
1-6 doses, 213 (17.4\%) discontinued after 7-12 doses, 152 (12.4\%) discontinued \\
after 13-18 doses, 188 (15.4\%) discontinued after 19-24 doses, 209 (17.1\%) \\
discontinued after 25-30 doses and 237 (19.4\%) discontinued after >30 doses. \\
Patients could have more than one reason for discontinuation/withdrawal recorded. \\
₹Medication changes were primarily to other DMTs. \\
\$Although PML was cited as the reason for discontinuation in only seven PML cases, \\
natalizumab was discontinued in all 18 cases upon suspicion of PML. Diagnosis was \\
made days to weeks after the last dose. \\
DMT, disease-modifying therapy; IS, immunosuppressant; JCV, JC virus; PML, \\
progressive multifocal leucoencephalopathy; TOP, Tysabri Observational Program.
\end{tabular}

seriousness criteria. The contract research organisation was notified of discrepancies for resolution with the site investigators.

Study endpoints were assessed uniformly across sites. To assure standardised examinations and consistent definitions for the EDSS Functional System (FS) scores, participating physicians were provided a copy of the interactive Neurostatus Training DVD-ROM, ${ }^{7}$ and Neurostatus certification was highly recommended. Investigators not previously certified were offered free-of-charge online certification (http://www.neurostatus.net). To reduce the risk of entry error with EDSS score reporting, the electronic case report form (CRF) calculated an EDSS score based on the Kurtzke FS and ambulation scores that were entered. The investigator either confirmed the calculated score or entered their own score with an explanation of why the proposed score was incorrect.

CRFs were designed to automatically generate queries for data inconsistencies, including data that were out of range or otherwise invalid. Data quality control procedures checked for consistency across data sets. Site-based verification and correction was used for residual data queries.

\section{Statistical analysis}

This study is designed to include at least 4500 patients. The sample size allows detection of a serious adverse event with an incidence of $1 / 1000$ with $95 \%$ probability. Enrolment started in July 2007, and patients will be followed for up to 10 years.

Descriptive statistics were used to summarise baseline characteristics and outcome measures. Efficacy and safety analyses, without imputation of missing data, were performed using the population of patients who provided a consent form, met inclusion and exclusion criteria and had received three or fewer doses of natalizumab at enrolment. 
Table 3 Summary of serious adverse events with an incidence $>1$

\begin{tabular}{|c|c|c|}
\hline Serious adverse event* & $\begin{array}{l}\text { All serious } \\
\text { adverse events }\end{array}$ & $\begin{array}{l}\text { Incidencet of serious } \\
\text { adverse events, } \mathrm{n}(\%)\end{array}$ \\
\hline Overall & $465 \ddagger$ & $388(8.0)$ \\
\hline Infection & 97 & $93(1.9)$ \\
\hline Not coded & 56 & $55(1.1)$ \\
\hline Hypersensitivity reaction & 26 & $26(0.5)$ \\
\hline Malignancy & 24 & $24(0.5)$ \\
\hline PML & 18 & $18(0.4)$ \\
\hline Abortion & 15 & $13(0.3)$ \\
\hline Thrombosis/embolism & 12 & $12(0.2)$ \\
\hline Elevated liver function tests & 7 & $7(0.1)$ \\
\hline Depression & 7 & $5(0.1)$ \\
\hline Herniated disc & 5 & $4(0.1)$ \\
\hline Epileptic seizure & 4 & $4(0.1)$ \\
\hline Panic attack & 4 & $4(0.1)$ \\
\hline Bladder disorder & 3 & $3(0.1)$ \\
\hline Migraine aggravated & 3 & $3(0.1)$ \\
\hline $\begin{array}{l}\text { Mood disorder not otherwise } \\
\text { specified }\end{array}$ & 3 & $3(0.1)$ \\
\hline Neurologic symptoms & 3 & $3(0.1)$ \\
\hline Suicide & 3 & $3(0.1)$ \\
\hline TIA & 3 & $3(0.1)$ \\
\hline Abdominal pain & 2 & $2(0.0)$ \\
\hline Anaemia & 2 & $2(0.0)$ \\
\hline Attempted suicide & 2 & $2(0.0)$ \\
\hline Back pain & 2 & $2(0.0)$ \\
\hline Conversion disorder & 2 & $2(0.0)$ \\
\hline Extravasation & 2 & $2(0.0)$ \\
\hline Fall & 2 & $2(0.0)$ \\
\hline Fracture femur & 2 & $2(0.0)$ \\
\hline Headache & 2 & $2(0.0)$ \\
\hline Haemorrhoidal crisis & 2 & $2(0.0)$ \\
\hline Hypertension & 2 & $2(0.0)$ \\
\hline $\begin{array}{l}\text { Immune reconstitution } \\
\text { inflammatory syndrome }\end{array}$ & 2 & $2(0.0)$ \\
\hline Menometrorrhagia & 2 & $2(0.0)$ \\
\hline Multiple drug overdose & 2 & $2(0.0)$ \\
\hline Myocardial infarction & 2 & $2(0.0)$ \\
\hline $\begin{array}{l}\text { Non-ST segment elevation } \\
\text { myocardial infarction }\end{array}$ & 2 & $2(0.0)$ \\
\hline Psychosis & 2 & $2(0.0)$ \\
\hline Psychosomatic disease & 2 & $2(0.0)$ \\
\hline Renal calculus & 2 & $2(0.0)$ \\
\hline Status epilepticus & 2 & $2(0.0)$ \\
\hline Uterine leiomyoma & 3 & $2(0.0)$ \\
\hline Vertigo & 2 & $2(0.0)$ \\
\hline
\end{tabular}

Annualised relapse rates were estimated using a negative binomial model. Time to EDSS progression and improvement and cumulative probabilities were analysed using the Kaplan-Meier method.

A repeated negative binomial model was used for a paired comparison of annualised relapse rate prenatalizumab and postnatalizumab treatment start. Associations between baseline factors and on-treatment annualised relapse rates were evaluated using a negative binomial model. A univariate analysis of each demographic and baseline disease characteristic with on-treatment annualised relapse rates was performed. Any variable with a $\mathrm{p}$ value $<0.25$ was considered in a multivariate negative binomial model. Baseline variables included in the adjusted negative binomial model were sex, relapse, EDSS score, disease duration, number of previous disease-modifying therapies (DMTs) used and treatment duration.

To further assess associations between baseline treatment history and on-treatment annualised relapse rates, patients were stratified into five treatment-history groups comprising therapies commercially available at the start of the study: therapy naïve, interferon $\beta-1$ (IFN) only, glatiramer acetate (GA) only, exposed to IFN and GA (either order), and a final group with any prior immunosuppressant (IS) use. A negative binomial regression model was used, adjusting for the baseline covariates described above.

Associations between baseline treatment history and postbaseline serious adverse events were evaluated using Pearson $\chi^{2}$ or Fisher exact tests and Poisson model. For this analysis, patients were stratified into three groups: therapy naïve, at least one prior DMT without prior IS use, and prior IS use.

\section{RESULTS}

\section{Patient disposition and baseline characteristics}

As of 1 December 2012, 4821 patients from 16 countries were enrolled in TOP. Table 1A,B summarises baseline characteristics. Differences between the AFFIRM and the TOP populations at baseline are described in table 1A. Table 2 summarises patient enrolment and disposition data. Overall, 77\% $(n=3703)$ were followed for at least 1 year, $52 \%(n=2496)$ for at least 2 years, $25 \%(n=1220)$ for at least 3 years and 10\% $(n=468)$ for at least 4 years. The median follow-up period was 26 months (range 1-69). Of the 4821 enrolled patients, all received at least one dose of natalizumab. The median duration of natalizumab therapy was 22 months (range 1-74).

Overall, 1222 of 4821 patients (25.3\%) discontinued natalizumab treatment and 740 of $4821(15.3 \%)$ withdrew from TOP; 523 patients discontinued natalizumab and withdrew from study follow-up. Patients may have discontinued natalizumab for more than one reason; the most common reasons included anti-JC virus (JCV) antibody positive status $(\mathrm{n}=277 ; 5.7 \%$ of all TOP patients), medication change $(n=247 ; 5.1 \%)$, insufficient efficacy $(n=229$; $4.8 \%)$ and patient decision $(n=171 ; 3.5 \%)$. Fifty-one patients discontinued due to serious adverse events. The most common reasons for study withdrawal included loss to follow-up $(n=121$; $2.5 \%)$, medication change $(n=116 ; 2.4 \%)$, patient decision $(\mathrm{n}=115 ; 2.4 \%)$, withdrawal of consent $(\mathrm{n}=110 ; 2.3 \%)$ and movement out of the area $(n=99 ; 2.1 \%)$.

Baseline characteristics were comparable between the 1222 patients who discontinued natalizumab treatment and the 3599 patients who remained on treatment, with the exception of a higher proportion of patients with prior IS use among those who discontinued $(18 \%)$ versus those who remained on therapy $(13 \%, \mathrm{p}<0.0001)$.

\section{Safety}

Table 3 summarises serious adverse events recorded at the time of data lock. Overall, $8.0 \%$ of patients experienced a serious adverse event and $2.6 \%$ experienced a serious adverse event that was considered related or possibly related to natalizumab. The most common serious adverse event was infection (incidence 1.9\%). Among the 97 serious infections, there were 18 herpes infections, 17 urinary tract infections and 16 pneumonias. Twelve of the 97 serious infections were identified as 
Figure 1 Annualised relapse rate on natalizumab therapy: per yearly interval over time $(n=4821)$. Error bars are excluded. indicate $95 \%$ Cls. Data beyond 5 years

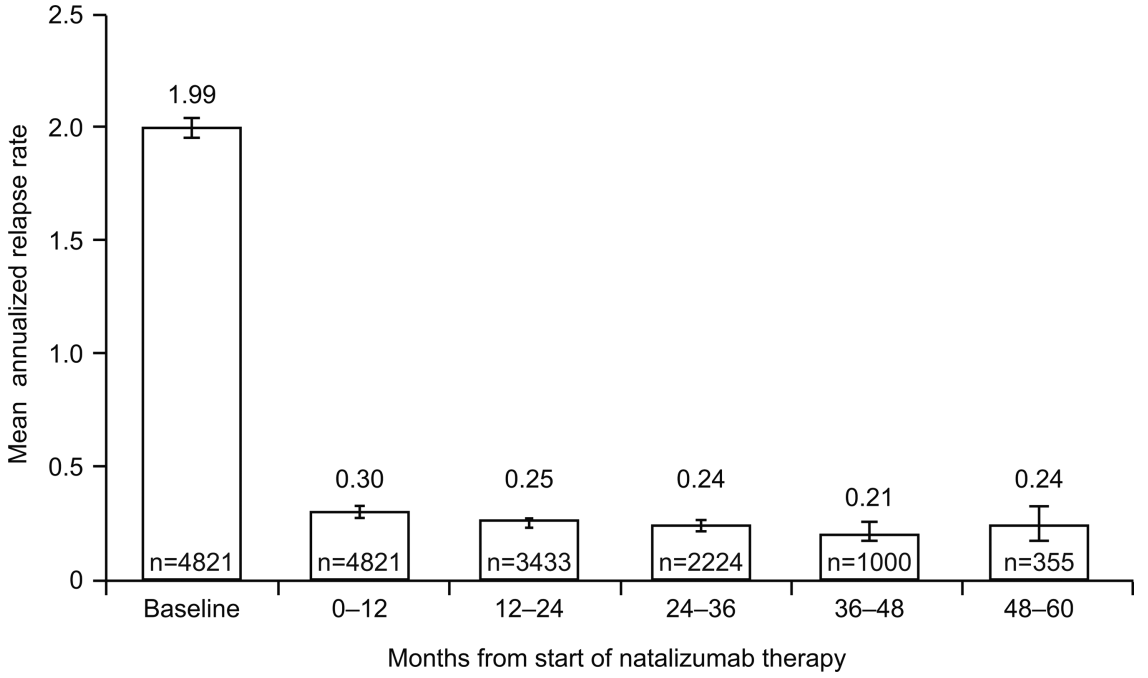

opportunistic; these included two herpes zoster, two herpes meningitides and two pneumonias, as well as one each of urinary tract infection, pyelonephritis, streptococcal meningitis, staphylococcal sepsis, encephalitis and Mycobacterium kansasii infection. The incidence of serious hypersensitivity reactions was $0.5 \%$, including anaphylaxis/anaphylactoid reactions $(0.2 \%)$.

Eighteen patients were diagnosed with progressive multifocal leucoencephalopathy (PML) after a median of 29 natalizumab doses (range 11-44). Fourteen of the 18 PML cases occurred in patients receiving natalizumab for $\geq 2$ years; four PML patients previously used IS (mitoxantrone in three cases; azathioprine in the fourth); 13 of the patients had received at least one DMT prior to natalizumab. The overall PML incidence rate was $0.4 \%$ (3.73/1000), with a $95 \%$ CI of 0.2 to 0.6 . Seven of the $18 \mathrm{PML}$ patients had confirmed positive anti-JCV antibody serostatus at least 6 months (6-15 months) prior to PML development; serostatus at least 6 months prior to PML development was unknown or not reported for 11 patients. At the time of this analysis (December 2012), 16 of the 18 patients (89\%) diagnosed with PML were alive.

There were 24 patients with 12 types of malignancies. Breast cancer was the most common, affecting seven patients (all female). Papillary thyroid cancer was diagnosed in three patients; cervical cancer, melanoma (one in situ) and leukaemia were each diagnosed in two patients; the other cancer diagnoses (rectal cancer, colon cancer, glioblastoma, basal cell carcinoma, kidney tumour, prostate cancer and testicular neoplasm) each occurred in one patient.

Nine deaths $(9 / 4821=0.2 \%)$ occurred during the study. One death was attributed to urosepsis (patient also had PML, which was ongoing at time of death), and there were three suicides, two fatal pulmonary emboli, one fatal drowning, one death due to autonomic nervous system imbalance and one death due to thermal burn.

\section{Relapses}

Overall, the annualised relapse rate (95\% CI) decreased from 1.99 (1.95 to 2.03 ) in the 12 months prior to baseline to 0.31 $(0.29$ to 0.32$)$ on natalizumab therapy $(p<0.0001)$. Relapse rates in each year of natalizumab exposure ranged from 0.21 to 0.30 (figure 1).

Significant associations between baseline disease characteristics and annualised relapse rates on natalizumab therapy were observed (figure 2). Lower mean on-therapy annualised relapse rates were associated with lower baseline EDSS scores, fewer zumab (figure 2A). When therapy history and baseline relapse status were analysed together, the lowest on-therapy annualised relapse rates were seen in patients with a history of only one relapse in the prior 12 months who were either therapy naïve or had received only one DMT prior to natalizumab; those with greater numbers of relapses and/or DMTs had higher rates $(0.16$ to 0.18 vs 0.23 to $0.40 ; \mathrm{p}<0.0001$ ) (figure $2 \mathrm{~B}$ ). While patients treated with natalizumab had significant reductions in annualised relapse rate regardless of treatment history, annualised relapse rates were lowest in patients who were therapy naïve at baseline and highest in those patients with prior IS use (figure 2C). In pairwise comparisons, annualised relapse rates were significantly different for the therapy-naïve group versus each of the other groups and for any of the comparisons of GA and/or IFN use groups versus the prior IS use group $(\mathrm{p}<0.05)$.

In the overall population, the cumulative probabilities of remaining relapse free by $1,2,3,4$ and 5 years on treatment were $78.9 \%, 67.1 \%, 56.3 \%, 50.0 \%$ and $44.4 \%$, respectively.

The 229 patients who discontinued natalizumab due to 'insufficient efficacy' had more severe disease than the overall TOP population prior to natalizumab treatment, as indicated by a higher median baseline EDSS score (4.5 vs 3.5) and a higher median baseline Multiple Sclerosis Severity Scale score (6.4 vs 4.9). Compared with the overall TOP population, a greater proportion of these patients relapsed during the first year in the study (45.4\% vs $18.1 \%)$. While antinatalizumab antibody status data were not collected routinely during the study, the presence of antinatalizumab antibodies was listed as a reason for discontinuing natalizumab for 44 of the 1222 patients (3.6\%) who discontinued treatment.

\section{Impairment and disability}

In the overall population, the mean (SD) EDSS score was 3.5 (1.62) in 4797 patients at baseline, $3.3(1.76 ; n=2064)$ after 1 year, $3.3(1.84 ; \mathrm{n}=1304)$ after 2 years, $3.3(1.84 ; \mathrm{n}=744)$ after 3 years and $3.3(1.92 ; n=325)$ after 4 years of observation.

Using the prespecified definitions, the cumulative probability of confirmed EDSS progression at 4 and 5 years was 14\%. The cumulative probability of confirmed EDSS improvement was $24 \%$ at 4 years and $27 \%$ at 5 years. Using the clinical trial definition of EDSS progression as defined above, the cumulative relapses in the prior year and fewer DMTs used prior to natali- 
Figure 2 Annualised relapse rate after initiation of natalizumab according to $(A)$ baseline characteristics, (B) combined baseline relapse status and number of prior disease-modifying therapies (DMTs) and (C) baseline treatment history. ${ }^{*} p$ Value from a negative binomial regression model adjusted for sex, baseline EDSS score $(<3.0$ vs $\geq 3.0)$, relapse status in the past year $(\leq 1$ vs $>1)$, prior DMT use $(0,1$ and $>1)$, disease duration ( $\geq 8$ vs $<8$ years) and treatment duration ( $\geq 3$ vs $<3$ years). $t p=0.697$ between comparator groups at baseline on the basis of a negative binominal model for baseline annualised relapse rate (adjusted for sex, baseline EDSS score ( $<3.0$ vs $\geq 3.0$ ), disease duration ( $<8$ vs $\geq 8$ years) and treatment duration $(<3$ vs $\geq 3$ years)). $\neq p<0.0001$ for difference among groups after treatment is from negative binomial model adjusted for sex, baseline relapse status ( 0 or 1 relapse vs $>1$ ), EDSS score $(<3.0$ vs $\geq 3.0)$, treatment duration ( $<3$ vs $\geq 3$ years) and disease duration ( $<8$ vs $\geq 8$ years). Error bars indicate $95 \%$ Cls. DMT,

disease-modifying therapy; EDSS, Expanded Disability Status Scale; GA, glatiramer acetate; IFN, interferon $\beta-1$; IS, immunosuppressant.
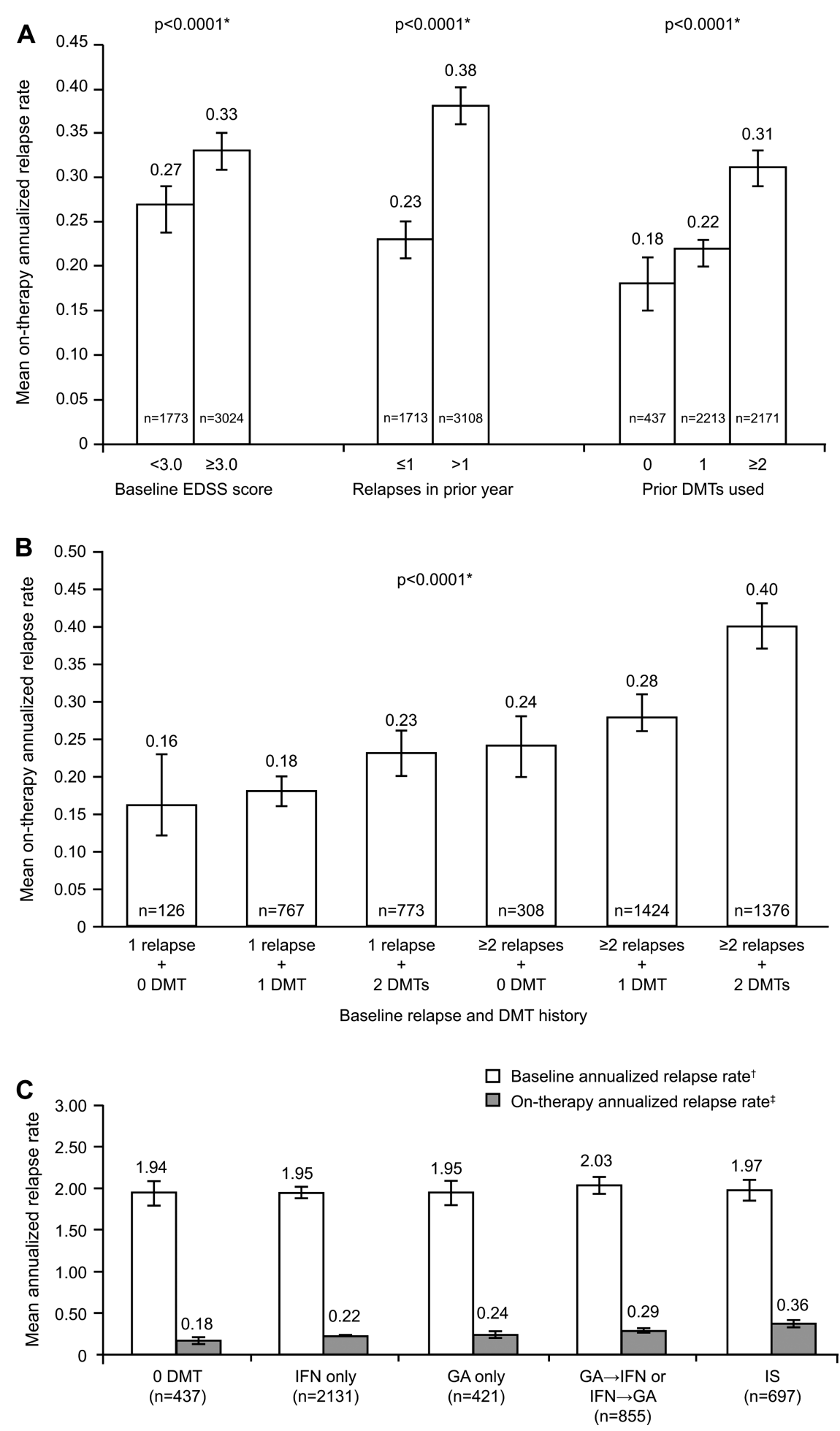

probability of confirmed EDSS progression at 5 years was $16 \%$ (figure 3A). The cumulative probability of improvement at 5 years in the subgroup of patients with baseline EDSS scores $\geq 2.0^{6}$ was $29 \%$ (figure 3B). The probability of confirmed EDSS improvement was significantly higher than the probability of confirmed worsening $(\mathrm{p}<0.0001)$.

It is noteworthy to emphasise that the rate of 12 -month confirmed EDSS progression, which might be informative for secondary progression, was not significantly different between the 229 patients who discontinued treatment due to insufficient efficacy' (median follow-up 13 months) as the stated reason for natalizumab cessation and the 3580 patients who remained on treatment (median follow-up 23 months): $6 \%$ and $4 \%$ of patients, respectively, had confirmed EDSS progression $(p=0.118)$.

\section{DISCUSSION}

In this interim analysis of the first 5 years of the TOP study, safety findings were consistent with previous studies of natalizumab, ${ }^{2} \quad 8^{8-10}$ and no new safety concerns were identified in a clinical practice setting. The most frequently reported serious adverse event in TOP to date was infection (1.9\%), which was also the most frequently reported serious adverse event (aside from MS relapse) among all patients receiving 
Figure 3 (A) Time from first natalizumab infusion to confirmed EDSS progression in overall study population. (B) Time from first natalizumab infusion to confirmed EDSS improvement in patients with baseline EDSS scores $\geq 2.0$. EDSS progression was defined as an increase, sustained for 6 months, of $\geq 0.5$ points from a baseline EDSS score $\geq 6.0$, of $\geq 1.0$ point from a baseline EDSS score of $\geq 1.0$ to $<6.0$ or of $\geq 1.5$ points from a baseline EDSS score of 0.0 . EDSS improvement was defined as a decrease, sustained for 6 months, of $\geq 1.0$ point from baseline EDSS score. EDSS, Expanded Disability Status Scale.
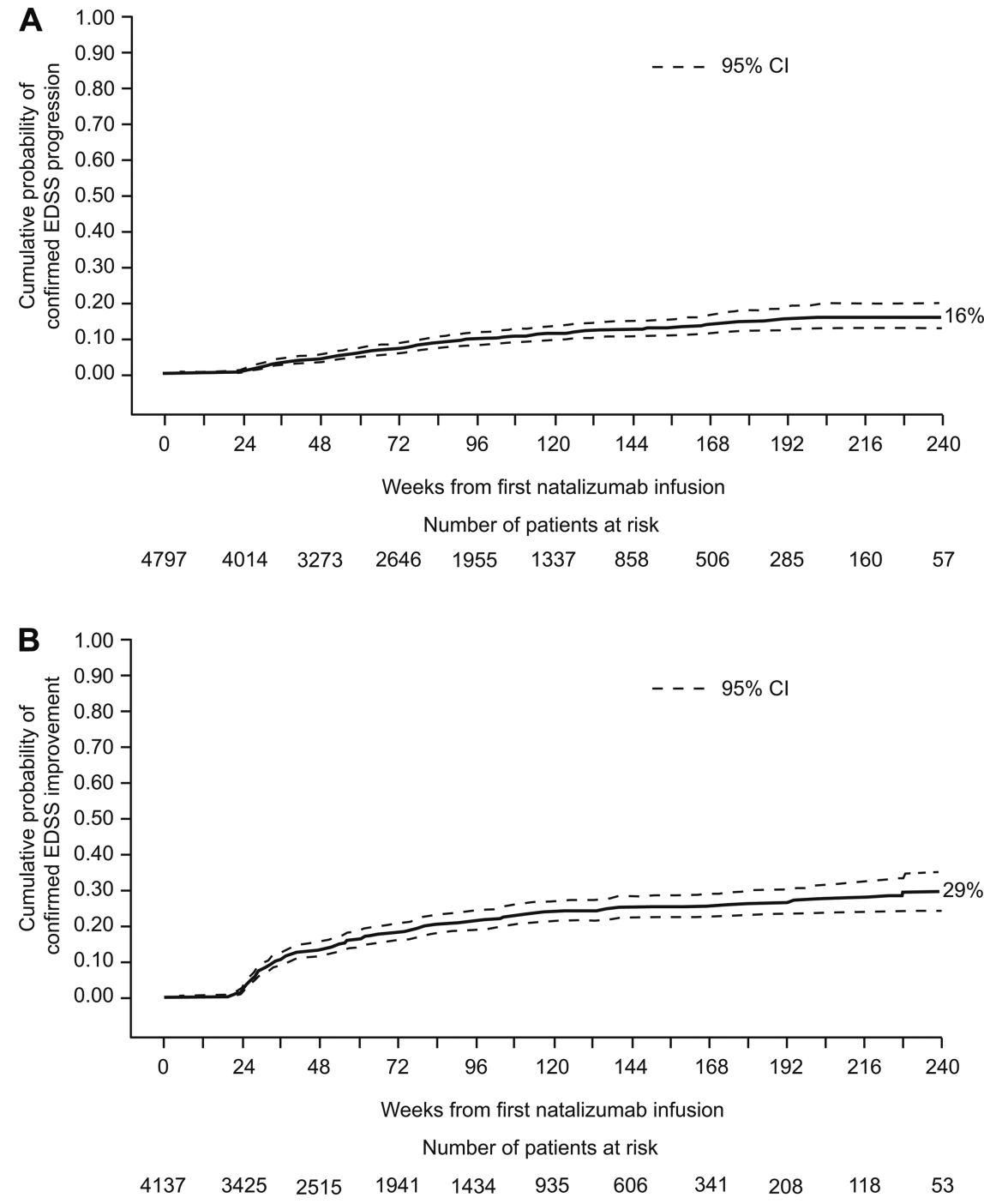

natalizumab in the phase 3 monotherapy trial (3.2\%). ${ }^{2} 11$ There were 17 reported cases of serious urinary tract infection $(0.4 \%)$ and 16 serious pneumonias $(0.3 \%)$ in TOP, compared with rates of $0.8 \%$ and $0.6 \%$, respectively, in natalizumab-treated patients in the phase 3 trial. $^{2} 11$ Opportunistic infections other than PML were reported with an incidence of $0.2 \%$ in TOP, which is consistent with the rate of $<1 \%$ observed in clinical trials. ${ }^{2} 11$

The overall PML incidence in TOP $(0.4 \%$ (3.73 cases $/ 1000$ patients)) is similar to the most recently reported risk in the natalizumab postmarketing setting (3.40 cases/1000 patients). ${ }^{12}$ Fourteen of 18 PML cases occurred in patients receiving natalizumab for $\geq 2$ years. In all seven cases with reported anti-JCV antibody serostatus at least 6 months prior to PML development, results were positive. These data are consistent with the known markedly increased risk of PML in anti-JCV antibody positive patients with a longer duration of natalizumab therapy. ${ }^{13}$ The risk of PML in patients who are anti-JCV antibody positive, have had prior IS use and have been exposed to natalizumab for $25-48$ months is estimated at 11.2 cases per $1000 .^{12}$ The risk in patients who are anti-JCV antibody negative is estimated at 0.1 cases per $1000^{12}$; in these patients, prior IS use and treatment duration do not affect PML risk as long as the patients remain anti-JCV antibody negative. Direct comparison of these risk estimates with those derived from TOP data cannot be made as anti-JCV antibody serostatus data were not collected throughout the protocol period. A recent protocol amendment has been approved to collect this information.

The observation that a significantly greater proportion of patients who discontinued natalizumab therapy had prior IS use compared with patients who remained on therapy suggests that decisions regarding treatment discontinuation were driven, at least in part, by PML risk factor assessment.

Rates of serious hypersensitivity reactions $(0.5 \%)$ and anaphylaxis or anaphylactoid reactions $(0.2 \%)$ in TOP were lower than the rates of $1.3 \%$ and $0.8 \%$, respectively, among natalizumab-treated clinical trial patients. ${ }^{2}$

Malignancies were described in 24 patients; breast cancer was the most common neoplasm, occurring at a rate consistent with the expected European incidence rate. ${ }^{14}$ Further follow-up in TOP will evaluate the risk of malignancy with longer natalizumab exposure.

On-treatment annualised relapse rate was significantly reduced and remained low after 5 years of natalizumab exposure. EDSS scores remained stable over time, and the probability of confirmed EDSS improvement was significantly higher than the probability of confirmed worsening $(p<0.0001)$. Even severely affected patients at baseline, namely patients with higher numbers of relapses, high EDSS scores and/or use of multiple prior therapies, achieved excellent clinical disease control, with an on-therapy annualised relapse rate $\leq 0.38$ for all subgroups. These findings accord with other observational studies and registries, in which significant reductions in 
annualised relapse rates were observed over 1-3 years of natalizumab treatment in clinical practice settings. ${ }^{15-17}$

In conclusion, this interim 5-year analysis of TOP data extends findings of randomised controlled pivotal trials by describing serious adverse event incidence in a large cohort of natalizumab-exposed patients with RRMS and demonstrates the low relapse probability and low confirmed EDSS progression events associated with natalizumab treatment. Unlike the AFFIRM population, in which the majority of patients were treatment naïve, ${ }^{2}$ the majority of patients in TOP $(\approx 90 \%)$ were previously treated with a DMT and/or IS therapy. Although relapse rates remained low across all groups on natalizumab, patients who started natalizumab treatment when they were therapy naïve, or with lower baseline EDSS scores or relapse rates, or who used fewer prior DMTs had lower on-therapy annualised relapse rates, suggesting there may be a lower clinical disease activity level when natalizumab treatment is initiated earlier in the disease course. TOP and other observational registries of natalizumab-treated patients will continue to generate valuable data on the long-term (5-10-year) safety and efficacy profile of natalizumab in a clinical practice setting.

\author{
Author affiliations \\ 'Department of Medicine, Melbourne Brain Centre at the Royal Melbourne Hospital, \\ University of Melbourne, Melbourne, Victoria, Australia \\ ${ }^{2}$ Department of Neurology, Box Hill Hospital, Monash University, Victoria, Victoria, \\ Australia \\ ${ }^{3}$ Departments of Neurology and Biomedicine, University Hospital Basel, Basel, \\ Switzerland \\ ${ }^{4}$ Department of Clinical Pharmacology and Epidemiology, Consorzio Mario Negri \\ Sud, Chieti, Italy \\ ${ }^{5}$ Department of Neuroscience and Sense Organs, University of Bari, Bari, Italy \\ ${ }^{6}$ Department of Neurology-Inflammatory Disorders of the Nervous System and \\ Neurooncology, University of Münster, Münster, Germany \\ ${ }^{7}$ Biogen Idec Inc, Cambridge, Massachusetts, USA
}

Acknowledgements The authors would like to acknowledge Amy Pace, SCD, of Biogen Idec Inc for her expert assistance with statistical analysis and interpretation; Dominic Paes, PhD, of Biogen Idec Inc, and Daniel Desgrandchamps, MD, formerly of Biogen Idec Inc, for their significant contributions to the study design, the start of the study and coordination of the initial 2 years of the study on behalf of Biogen Idec Inc; and Samir Mechati of Rodanotech Sarl for his contributions to database design and customisation. Katherine Hauswirth, RN, MSN, from Infusion Communications wrote the first draft of the manuscript based on input from authors, and Jackie Cannon from Infusion Communications copyedited and styled the manuscript per journal requirements.

Collaborators HB, LK, FP, MT and HW are TOP Steering Committee members (a full list of the Investigators can be found online as supplementary material).

Contributors HB contributed to the study design, patient recruitment, data collection, analysis and interpretation of data, and drafting and revising of the manuscript; he takes full responsibility for the finished article, access to any data, and controlled the decision to publish. LK contributed to the study design, analysis and interpretation of data, and drafting and revising of the manuscript. FP contributed to the analysis and interpretation of data and drafting and revising of the manuscript. MT contributed to the study design, patient recruitment, data collection, analysis and interpretation of data, and drafting and revising of the manuscript. HW contributed to the funding, study design, analysis and interpretation of data, and drafting and revising of the manuscript. RNP contributed to the analysis and interpretation of data and drafting and revising of the manuscript. AZ contributed to the statistical analysis, analysis and interpretation of data, and drafting and revising of the manuscript. CH contributed to the study design, analysis and interpretation of data, and drafting and revising of the manuscript. SB contributed to the study design, patient recruitment, data collection, statistical analysis, analysis and interpretation of data, and drafting and revising of the manuscript.

Funding This study was supported by Biogen Idec Inc, which also provided funding for editorial support in the development of this manuscript. Biogen Idec reviewed the manuscript and provided feedback on the manuscript. The authors had full editorial control of the manuscript and provided their final approval of all content.

Competing interests HB has been a member of scientific advisory boards for Biogen Idec, Merck Serono, Novartis and sanofi-aventis; has received conference travel support from Biogen Idec, Merck Serono, Novartis and sanofi-aventis; has received fees for steering committees for trials conducted by Biogen Idec, Merck
Serono and Novartis; has received awards and grants including the National Health and Medical Research Council (NHMRC) Career Development Award, NHMRC Project Grants, NHMRC Centre of Excellence Award, Australian Research Council Linkage Grant and National MS Society (USA) Project Grant; is on the editorial board of Multiple Sclerosis International and Multiple Sclerosis and Related Disorders; and has received research support from Biogen Idec, Merck Serono and Novartis, as honorary chair of the MSBase Foundation. LK has received research support from Acorda, Actelion, Allozyne, BaroFold, Bayer HealthCare, Bayer Schering, Bayhill, Biogen Idec, Boehringer Ingelheim, Elan, Genmab, Glenmark, GlaxoSmithKline, Merck Serono, MediciNova, Novartis, sanofi-aventis, Santhera, Shire, Roche, Teva, UCB, Wyeth, Swiss MS Society, Swiss National Research Foundation, European Union, Gianni Rubatto Foundation, and the Novartis and Roche Research Foundations. FP has received consulting fees and honoraria from Biogen Idec. MT has been a consultant for and received speaker honoraria from Bayer Schering, Biogen Idec, Novartis and sanofi-aventis and has received research grants from Biogen Idec and Merck Serono. HW has received honoraria from Bayer, Biogen Idec/Elan, Medac, Merck Serono, Novo Nordisk, sanofi-aventis, Schering and Teva; has been a consultant for Bayer Vital/Schering, Biogen Idec, Medac, Merck Serono, Novartis, Novo Nordisk, sanofi-aventis and Teva; and has received research support from Bayer, Biogen Idec/Elan, Medac, Merck Serono, Novo Nordisk, sanofi-aventis, Schering and Teva. RNP, AZ, CH and SB are employees of Biogen Idec.

Ethics approval Ethics committees for all participating study centres.

Provenance and peer review Not commissioned; externally peer reviewed.

Open Access This is an Open Access article distributed in accordance with the Creative Commons Attribution Non Commercial (CC BY-NC 3.0) license, which permits others to distribute, remix, adapt, build upon this work non-commercially, and license their derivative works on different terms, provided the original work is properly cited and the use is non-commercial. See: http://creativecommons.org/ licenses/by-nc/3.0/

\section{REFERENCES}

1 Rudick RA, Sandrock A. Natalizumab: $\alpha 4$-integrin antagonist selective adhesion molecule inhibitors for MS. Expert Rev Neurother 2004;4:571-80.

2 Polman $\mathrm{CH}, \mathrm{O}^{\prime}$ Connor PW, Havrdova E, et al. A randomized, placebo-controlled trial of natalizumab for relapsing multiple sclerosis. N Engl J Med 2006;354:899-910.

3 Hutchinson M, Kappos L, Calabresi PA, et al. The efficacy of natalizumab in patients with relapsing multiple sclerosis: subgroup analyses of AFFIRM and SENTINEL. J Neurol 2009;256:405-15.

4 Roxburgh RH, Seaman SR, Masterman T, et al. Multiple Sclerosis Severity Score: using disability and disease duration to rate disease severity. Neurology 2005;64:1144-51.

5 Rudick RA, Stuart WH, Calabresi PA, et al. Natalizumab plus interferon beta-1a for relapsing multiple sclerosis. N Engl J Med 2006;354:911-23.

6 Phillips JT, Giovannoni G, Lublin FD, et al. Sustained improvement in Expanded Disability Status Scale as a new efficacy measure of neurological change in multiple sclerosis: treatment effects with natalizumab in patients with relapsing multiple sclerosis. Mult Scler 2011;17:970-9.

7 Kappos L, Wu S, eds. Interactive Training DVD-ROM for a standardised, quantified neurological examination and assessment of Kurtzke's Functional Systems and Expanded Disability Status Scale in Multiple Sclerosis. Basel, Switzerland: University Hospital Basel Department of Neurology, 2007.

8 O'Connor PW, Goodman AD, Kappos L, et al. Updated efficacy and safety of natalizumab in patients who participated in the STRATA study [abstract]. Mult Scler 2010;16(Suppl 10):S162.

9 Bozic C, Cristiano LM, Hyde R, et al. Utilisation and safety of natalizumab in patients with relapsing multiple sclerosis [abstract]. Mult Scler 2010;16(Suppl 10):S315.

10 Kappos L, Bates D, Edan G, et al. Natalizumab treatment for multiple sclerosis: updated recommendations for patient selection and monitoring. Lancet Neurol 2011;10:745-58

11 TYSABRI (natalizumab) injection. Full prescribing information. http://www.tysabri. com/pdfs/161061-13_PI.pdf (accessed 5 Aug 2013).

12 Biogen Idec Medlnfo. https://medinfo.biogenidec.com (accessed 9 Dec 2013)

13 Bloomgren G, Richman S, Hotermans $C$, et al. Risk of natalizumab-associated progressive multifocal leukoencephalopathy. N Engl J Med 2012;366:1870-80

14 Ferlay J, Steliarova-Foucher E, Lortet-Tieulent J, et al. Cancer incidence and mortality patterns in Europe: estimates for 40 countries in 2012. Eur J Cancer 2013;49:1374-403.

15 Putzki N, Yaldizli 0, Bühler R, et al. Natalizumab reduces clinical and MRI activity in multiple sclerosis patients with high disease activity: results from a multicenter study in Switzerland. Eur Neurol 2010;63:101-6.

16 Sangalli F, Moiola L, Bucello S, et al. Efficacy and tolerability of natalizumab in relapsing-remitting multiple sclerosis patients: a post-marketing observational study. Neurol Sci 2011;31(Suppl 3):299-302.

17 Kallweit $U$, Jelcic I, Braun $N$, et al. Sustained efficacy of natalizumab in the treatment of relapsing-remitting multiple sclerosis independent of disease activity and disability at baseline: real-life data from a Swiss cohort. Clin Neuropharmacol 2012;35:77-80 